\title{
An improved beam element for beams with variable axial parameters
}

\author{
Peng $\mathrm{He}^{*}$, Zhansheng Liu and Chun Li \\ School of Energy Science and Engineering, Harbin Institute of Technology, Harbin, Heilongjiang, China
}

Received 21 April 2012

Revised 4 December 2012

Accepted 14 January 2013

\begin{abstract}
The traditional beam element was improved to consider the variable axial parameters. The variable axial parameters were formulated in terms of a power series, and the general forms of elementary mass and stiffness matrices which depend on the power order were derived. The mass and stiffness matrices of the improved beam element were obtained in terms of an elementary matrix series. The beam elements for various tapered beams and a beam under linearly axial temperature distribution were derived. The vibrations of the beams with various taper shapes were studied and the variations of natural frequencies and modal shapes were investigated. A uniform beam under linearly axial temperature distribution was modeled and studied. The influences of axial temperature difference on the natural frequencies and modal shapes were investigated. Results show that the improved beam element could consider the variable axial parameters of beam conveniently.
\end{abstract}

Keywords: Variable axial parameters, transverse vibration, tapered beam, temperature, finite element method

\section{Introduction}

Many beams in engineering have variable axial parameters. Tapered beam, which has variable axial geometric parameters, is a typical case. Moreover, the beam under non-uniform axial temperature distribution has variable material mechanical parameters. The variable axial parameters bring obstacles in vibration analysis of these beams. In order to take the variable axial parameters into account conveniently, this work is motivated to improve the traditional beam element.

The transverse vibration of tapered beams has been investigated in many papers. A number of attentions were paid to the analytical solutions of some particular tapered beams. Based on the Frobenius method, Naguleswaran [1-3] derived the solutions for the transverse vibration of linearly tapered, double tapered and convex parabolic tapered beams. Abrate [4] transformed the motion equation of non-uniform beam into a uniform case. Acuiello and Ercolano [5] applied Bessel function to solve the motion equation of tapered beam. Zhou and Cheung [6,7] derived the static beam functions in vibration analysis of a type of tapered Euler-Bernoulli beam and Timoshenko beam. $\mathrm{Wu}$ et al. [8,9] adopted the combined analytical and numerical method to investigate the transverse vibration of a tapered Euler-Bernoulli beam. Ece et al. [10] derived an analytical solution for the vibration of an Euler-Bernoulli beam with exponentially varying width and constant height. Caruntu [11,12] studied the vibration of a tapered beam with parabolic thickness variation by Jacobi polynomials and by hypergeometric functions. Firouz-Abadi et al. [13] studied the tapered beam by an approximate analytical method. Hsu et al. [14] applied the Adomian modified decomposition method for a non-uniform Euler-Bernoulli beam. Based on Taylor series expansion, Kaya [15] studied a

*Corresponding author: Peng He, School of Energy Science and Engineering, Harbin Institute of Technology, Harbin 150001, Heilongjiang, China. Tel.: +86 04518641 3794; Fax: +86 04518641 3242; E-mail: hepeyfly@foxmail.com. 

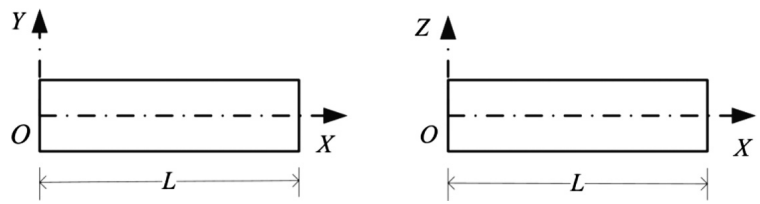

Fig. 1. The coordinate system of beam.

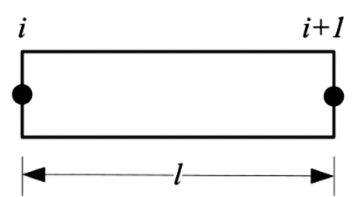

Fig. 2. Schematic of a simple Timoshenko beam element.

rotating tapered Timoshenko beam by differential transformation method. It can be seen that the analytical methods can solve the vibration problems of some particular tapered beams well, while the general analytical solution for various tapered beams is still missing. Many papers were focused on the finite element method for tapered beams. Klein [16] analyzed the vibration of a non-uniform beam by taking advantage of finite element method. To [17, 18] investigated the vibration of a linearly tapered Euler-Bernoulli beam and Timoshenko beam by finite element method. The linearly tapered beam elements were applied in rotor dynamics analysis [19-21]. Cleghorn and Tanbark [22] proposed a linearly tapered Timoshenko beam element. Some works [23,24] solved the governing equation by the Frobenius method and formulated a beam element for a rotating tapered beam. Based on the solution of static differential equation and cubic polynomial function, Gunda and Ganguli [25,26] derived a rational shape function for a tapered Euler-Bernoulli beam element. Attarnejad and Shahba [27] derived a set of new shape functions by a power series for a tapered Euler-Bernoulli beam element. Yardimoglu [28] proposed a beam element for a rotating tapered Timoshenko beam with equal strength. Shahba et al. [29] formulated a beam element for the vibration of axially functionally graded tapered Euler-Bernoulli beams. The published tapered beam elements are only focused on some particular types of tapered beams and massive derivations are needed to formulate the element matrices. When the beam tapered shape changes, it still needs massive derivations, which is not convenient for various tapered beams. In order to model the various tapered beams by finite element method conveniently, this work is intended to improve the traditional beam element.

It is known that the elastic moduli of most metals decrease with the increase of temperature. The vibration characteristics of the beam under axial temperature distribution will be influenced. Kimball and Lovell [30] investigated the variation of Young's modulus with temperature through the variations of natural frequencies. Liu et al. [31] studied the influence of temperature distribution on the critical speeds of a gas turbine rotor. For a beam under nonuniform axial temperature distribution, the Young's modulus varies along the axial direction, which has influence on the transverse vibration of beam. In order to consider the influence of axial temperature distribution, the traditional beam element should be improved to take the variation of Young's modulus into account.

Note that many finite element packages can be applied to study the vibration of beams with variable axial parameters. The commercial package ANSYS is a widely applied one, while it only offers a linearly tapered beam element [32], which can not model the beams with various taper shapes well. Of course, the tapered beam can be modeled by $3 \mathrm{D}$ elements and the beam under non-uniform axial temperature distribution can also be analyzed by $3 \mathrm{D}$ thermal-mechanical elements in commercial packages. However, much preprocess work is needed for three dimensional modeling and meshing, which is not convenient as the $1 \mathrm{D}$ beam elements.

By adding rotary inertia and shear effect into Euler-Bernoulli beam, Timoshenko beam is more accurate than Euler-Bernoulli beam, especially for thick beams. In this study, the simple Timoshenko beam element will be improved to consider the variable axial parameters, and it can also be reduced to Euler-Bernoulli beam element. The variations of axial parameters are formulated in terms of a power series. The general forms of elementary matrices which depend on the power order are derived, with which the beam element matrices can be obtained. The beams with various tapered shapes and a beam under linearly axial temperature distribution are modeled and studied by the improved beam element.

\section{Finite modeling of beams with variable parameters}

Considering a beam as shown in Fig. 1, only the vibration in $O X Y$ plane is concerned in this work, while the vibration in $O X Z$ can be studied by the same way. 
A simple Timoshenko beam element with nodes $i$ and $i+1$ is shown in Fig. 2. The degrees of freedom of the element are $\left\{v_{i}, \theta_{i}, v_{i+1}, \theta_{i+1}\right\}^{\mathrm{T}}$. Where $v$ is the lateral displacement, $\theta$ indicates the rotation of cross section. The shear deformation $\psi$ relates to $v$ and $\theta$ :

$$
\psi=\frac{\partial v}{\partial x}-\theta
$$

The kinetic energy of the element is the combination of lateral translation and rotation:

$$
T_{e}=\frac{1}{2} \int_{0}^{l} \rho A \dot{v}^{2} d s+\frac{1}{2} \int_{0}^{l} \rho I_{z} \dot{\theta}^{2} d s
$$

The potential energy of the element includes the bending and shearing effect:

$$
U_{e}=\frac{1}{2} \int_{0}^{l} E I_{z}(\partial v / \partial s)^{2} d s+\frac{1}{2} \int_{0}^{l}(G A / \chi) \psi^{2} d s
$$

Where $l$ is the length of the element, $\rho$ is the density of the beam material, $A$ is the area of the cross section, $I_{z}$ is the second moment inertia of cross section, $E$ and $G$ are the Young's modulus and shear modulus, respectively. $\chi$ is the shear factor which depends on the shape of the cross section.

Define the parameter $\phi$ :

$$
\phi=12 E I_{z} \chi /\left(G A l^{2}\right)
$$

The lateral displacement $v$ and rotation $\theta$ in the element can be written as:

$$
\begin{aligned}
& v=\mathbf{N}_{1}\left\{\begin{array}{llll}
v_{i} & \theta_{i} & v_{i+1} & \theta_{i+1}
\end{array}\right\}^{\mathrm{T}} \\
& \theta=\mathbf{N}_{2}\left\{\begin{array}{lllll}
v_{i} & \theta_{i} & v_{i+1} & \theta_{i+1}
\end{array}\right\}^{\mathrm{T}}
\end{aligned}
$$

According to Eq. (1), the shear deformation in the element can be written as:

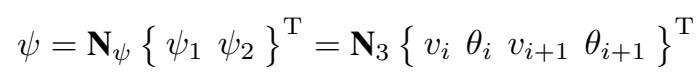

Here $\mathbf{N}_{1}$ and $\mathbf{N}_{2}$ are the interpolation functions of lateral displacement and cross section rotation respectively.

$$
\begin{aligned}
& \mathbf{N}_{1}=\left[\begin{array}{llll}
N_{11} & N_{12} & N_{13} & N_{14}
\end{array}\right] \\
& \mathbf{N}_{2}=\left[\begin{array}{llll}
N_{21} & N_{22} & N_{23} & N_{24}
\end{array}\right]
\end{aligned}
$$

The detailed formulations of $\mathbf{N}_{1}$ and $\mathbf{N}_{2}$ are listed in appendix. The interpolation function $\mathbf{N}_{3}$ relates to $\mathbf{N}_{1}$ and $\mathbf{N}_{2}$ :

$$
\mathbf{N}_{3}=\mathbf{N}_{2}-\frac{\mathrm{d}}{\mathrm{d} s} \mathbf{N}_{1}
$$

The lateral translation mass matrix $\mathbf{M}_{t}$ and the rotation mass matrix $\mathbf{M}_{r}$ can be written as:

$$
\begin{aligned}
& \mathbf{M}_{t}=\rho A \int_{0}^{l} \mathbf{N}_{1}^{\mathrm{T}} \mathbf{N}_{1} d s \\
& \mathbf{M}_{r}=\rho I_{z} \int_{0}^{l} \mathbf{N}_{2}^{\mathrm{T}} \mathbf{N}_{2} d s
\end{aligned}
$$

And the bending stiffness matrix $\mathbf{K}_{b}$ and shear stiffness $\mathbf{K}_{s}$ are:

$$
\mathbf{K}_{b}=E I_{z} \int_{0}^{l}\left(\mathrm{~d} \mathbf{N}_{2} / \mathrm{d} s\right)^{\mathrm{T}}\left(\mathrm{d} \mathbf{N}_{2} / \mathrm{d} s\right) \mathrm{d} s
$$




$$
\mathbf{K}_{s}=12 E I_{z} /\left(\phi l^{2}\right) \int_{0}^{l} \mathbf{N}_{3}^{\mathrm{T}} \mathbf{N}_{3} \mathrm{~d} s
$$

The mass matrix and stiffness matrix of the simple Timoshenko beam element can be obtained:

$$
\begin{aligned}
\mathbf{M}_{e} & =\mathbf{M}_{t}+\mathbf{M}_{r} \\
\mathbf{K}_{e} & =\mathbf{K}_{b}+\mathbf{K}_{s}
\end{aligned}
$$

Note that when the mass matrix only has the lateral transverse portion and the parameter $\phi$ relates to shear factor is zero, the simple Timoshenko beam element can be reduced to an Euler-Bernoulli beam element:

$$
\begin{aligned}
\phi & =0 \\
\mathbf{M}_{e} & =\mathbf{M}_{t} \\
\mathbf{K}_{e} & =\mathbf{K}_{b}
\end{aligned}
$$

In the formulations of traditional beam element, the parameters, such as $(\rho A)$ in $\mathbf{M}_{t},\left(\rho I_{z}\right)$ in $\mathbf{M}_{r},\left(E I_{z}\right)$ in $\mathbf{K}_{b}$ and $12 E I_{z} /\left(\phi l^{2}\right)$ in $\mathbf{K}_{s}$, are all constant.

\subsection{Finite element with variable axial parameters}

For a beam with variable axial parameters, the parameters $(\rho A)(s),\left(\rho I_{z}\right)(s),\left(E I_{z}\right)(s)$ and $\left(12 E I_{z} /\left(\phi l^{2}\right)\right)(s)$ are varying with the axial coordinate $s$. The axial parameters can have different variation forms, while they can be formulated in terms of a power series or be expanded into Taylor series.

$$
\begin{gathered}
(\rho A)(s)=(\rho A)_{0}+(\rho A)_{1}(s / l)+\ldots+(\rho A)_{m_{1}}(s / l)^{m_{1}}=\sum_{n=0}^{m_{1}}(\rho A)_{n}(s / l)^{n} \\
\left(\rho I_{z}\right)(s)=\left(\rho I_{z}\right)_{0}+\left(\rho I_{z}\right)_{1}(s / l)+\ldots+\left(\rho I_{z}\right)_{m_{2}}(s / l)^{m_{2}}=\sum_{n=0}^{m_{2}}\left(\rho I_{z}\right)_{n}(s / l)^{n} \\
\left(E I_{z}\right)(s)=\left(E I_{z}\right)_{0}+\left(E I_{z}\right)_{1}(s / l)+\ldots+\left(E I_{z}\right)_{m_{3}}(s / l)^{m_{3}}=\sum_{n=0}^{m_{3}}\left(E I_{z}\right)_{n}(s / l)^{n} \\
\left(\frac{12 E I_{z}}{\phi l^{2}}\right)(s)=\left(\frac{12 E I_{z}}{\phi l^{2}}\right)_{0}+\left(\frac{12 E I_{z}}{\phi l^{2}}\right)_{1}(s / l)+\ldots+\left(\frac{12 E I_{z}}{\phi l^{2}}\right)_{m_{4}}(s / l)^{m_{4}}=\sum_{n=0}^{m_{4}}\left(\frac{12 E I_{z}}{\phi l^{2}}\right)_{n}(s / l)^{n}
\end{gathered}
$$

Where $n$ is a nonnegative integer, which relates to the series order. $m_{1}, m_{2}, m_{3}$ and $m_{4}$ are the highest orders of each series. $(\rho A)_{n},\left(\rho I_{z}\right)_{n},\left(E I_{z}\right)_{n}$ and $\left(12 E I_{z} /\left(\phi l^{2}\right)\right)_{n}$ are constants for a definite order $n$.

Taking Eqs (16)-(19) into Eqs (9)-(12) respectively, the mass matrices and stiffness matrices of the element can be written as:

$$
\begin{aligned}
& \mathbf{M}_{t}=\sum_{n=0}^{m_{1}}(\rho A)_{n} \int_{0}^{l}(s / l)^{n} \mathbf{N}_{1}^{\mathrm{T}} \mathbf{N}_{1} \mathrm{~d} s=\sum_{n=0}^{m_{1}}(\rho A)_{n} \mathbf{M}_{t n} \\
& \mathbf{M}_{r}=\sum_{n=0}^{m_{2}}\left(\rho I_{z}\right)_{n} \int_{0}^{l}(s / l)^{n} \mathbf{N}_{2}^{\mathrm{T}} \mathbf{N}_{2} \mathrm{~d} s=\sum_{n=0}^{m_{2}}\left(\rho I_{z}\right)_{n} \mathbf{M}_{r n} \\
& \mathbf{K}_{b}=\sum_{n=0}^{m_{3}}\left(E I_{z}\right)_{n} \int_{0}^{l}(s / l)^{n}\left(\mathrm{~d} \mathbf{N}_{2} / \mathrm{d} s\right)^{\mathrm{T}}\left(\mathrm{d} \mathbf{N}_{2} / \mathrm{d} s\right) \mathrm{d} s=\sum_{n=0}^{m_{3}}\left(E I_{z}\right)_{n} \mathbf{K}_{b n} \\
& \mathbf{K}_{s}=\sum_{n=0}^{m_{4}}\left(12 E I_{z} /\left(\phi l^{2}\right)\right)_{n} \int_{0}^{l}(s / l)^{n} \mathbf{N}_{3}^{\mathrm{T}} \mathbf{N}_{3} \mathrm{~d} s=\sum_{n=0}^{m_{4}}\left(12 E I_{z} /\left(\phi l^{2}\right)\right)_{n} \mathbf{K}_{s n}
\end{aligned}
$$



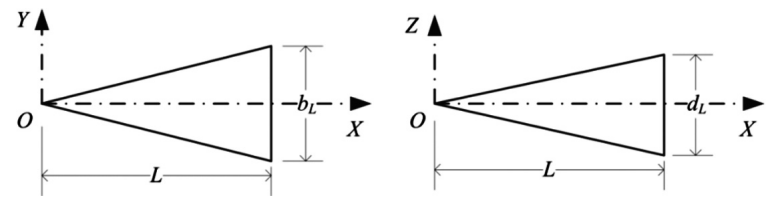

Fig. 3. Coordinate system of a complete wedge beam.
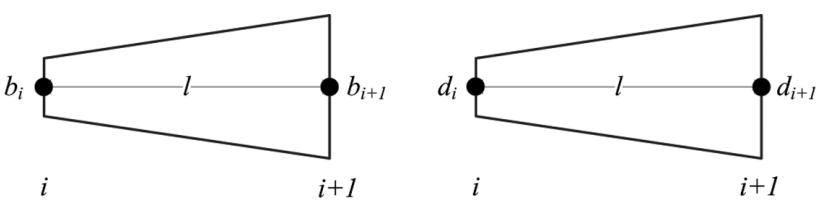

Fig. 4. Schematic of a tapered beam element with varying width and depth.

Where $\mathbf{M}_{t n}=\int_{0}^{l}(s / l)^{n} \mathbf{N}_{1}^{\mathrm{T}} \mathbf{N}_{1} d s, \mathbf{M}_{r n}=\int_{0}^{l}(s / l)^{n} \mathbf{N}_{2}^{\mathrm{T}} \mathbf{N}_{2} d s, \mathbf{K}_{b n}=\int_{0}^{l}(s / l)^{n}\left(d \mathbf{N}_{2} / d s\right)^{\mathrm{T}}\left(d \mathbf{N}_{2} / d s\right) d s$ and $\mathbf{K}_{s n}=\int_{0}^{l}(s / l)^{n} \mathbf{N}_{3}^{\mathrm{T}} \mathbf{N}_{3} d s$ are the elementary matrices which depend on the order $n$. They are listed in Appendix in detail.

The mass and stiffness matrices of the improved beam element can be obtained by Eqs (20)-(23). It can be seen that the matrices of the improved beam element are the superposition of an elementary matrix series, which relate to the series of variable axial parameters.

With the improved beam element, the beams with different types of variable axial parameters can be modeled and analyzed. The improved beam element can be applied by two steps:

(a) Formulate the variable axial parameters in terms of a power series.

The variation of the axial parameters in the beam element should be formulated in terms of a power series at first. In some cases, the variable parameters can be written in terms of a power series directly. Taking a double linearly tapered beam with rectangular cross section for example, the variation of area and second moment inertia of cross section can be formulated in terms of a power series directly. While in many cases, the variations can not be written as a power series directly and they can be expanded into Taylor series. As the Taylor series is an infinite series, the truncation order of Taylor expansion should be configured in this case.

(b) Generate the mass and stiffness matrices of beam element.

With the power series of the variable axial parameters, the mass and stiffness matrices can be obtained by Eqs (20)-(23) with the elementary matrices.

It can be seen that the improved beam element is not limited to any particular variation of axial parameters. Once the variations of axial parameters are formulated or expanded in terms of a power series, the element mass and stiffness matrices can be obtained. Since most of the variations can be expressed or expanded into a power series, the improved beam element can model the beams with many types of axial parameters variations. Moreover, the improved beam element can also be applied with Timoshenko beam theory and Euler-Bernoulli beam theory. However, it is clear that the improved beam element depends on the power series forms of the variable axial parameters, which needs some work to formulate the variations of axial parameters in terms of a power series ahead.

In order to illustrate the application of the improved beam element, the tapered beam with various tapered shapes and a beam under linearly axial temperature distribution are to be modeled and studied.

\subsection{Tapered beams}

Considering a complete tapered wedge beam, as illustrated in Fig. 3, the variations of width $b$ and depth $d$ can be written as:

$$
\begin{aligned}
& b=b_{L}(x / L)^{t b} \\
& d=d_{L}(x / L)^{t d}
\end{aligned}
$$

Where $b_{L}$ and $d_{L}$ are the width and depth of the large end, respectively. $t b$ and $t d$ are the tapered orders of width and depth, respectively.

The tapered wedge beam can be modeled by the improved beam element. For an element as illustrated in Fig. 4 , the variations of width $b$ and depth $d$ in the beam element can be written as:

$$
b=b_{i}\left[\left(\left(\left(b_{i+1}\right)^{\frac{1}{t b}}-\left(b_{i}\right)^{\frac{1}{t b}}\right) /\left(b_{i}\right)^{\frac{1}{t b}}\right)(s / l)+1\right]^{t b}=b_{i}[(\Delta b)(s / l)+1]^{t b}
$$




$$
d=d_{i}\left[\left(\left(\left(d_{i+1}\right)^{\frac{1}{t d}}-\left(d_{i}\right)^{\frac{1}{t d}}\right) /\left(d_{i}\right)^{\frac{1}{t d}}\right)(s / l)+1\right]^{t d}=d_{i}[(\Delta b)(s / l)+1]^{t d}
$$

Where $\Delta b=\left(\left(b_{i+1}\right)^{1 / t b}-\left(b_{i}\right)^{1 / t b}\right)\left(\left(b_{i}\right)^{1 / t b}\right)^{-1}$ and $\Delta d=\left(\left(d_{i+1}\right)^{1 / t d}-\left(d_{i}\right)^{1 / t d}\right)\left(\left(d_{i}\right)^{1 / t d}\right)^{-1}$.

The tapered orders $t b$ and $t d$ are different for various tapered beam element. They can be classified into integer tapered order and fraction tapered order. For a beam with integer taper order, the width and depth can be written as a power series directly:

$$
\begin{aligned}
& b=\mathbf{C}_{\mathbf{b}}\left\{\begin{array}{lllll}
1 & s / l & \ldots & (s / l)^{n} \ldots(s / l)^{t b}
\end{array}\right\}^{\mathrm{T}} \\
& d=\mathbf{C}_{\mathbf{d}}\left\{\begin{array}{lllll}
1 & s / l & \ldots & \ldots(s / l)^{n} \ldots(s / l)^{t d}
\end{array}\right\}^{\mathrm{T}}
\end{aligned}
$$

Where the coefficients vector $\mathbf{C}_{\mathbf{b}}$ and $\mathbf{C}_{\mathbf{d}}$ are:

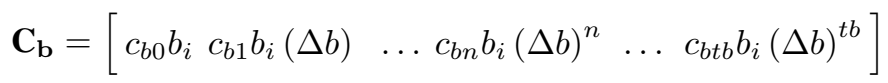

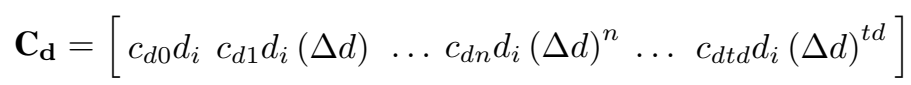

Here the parameters $c_{b n}$ and $c_{d n}$ are binomial coefficients.

For fraction taper orders, the variations of width $b$ and depth $d$ can not be written in terms of a power series directly. However, they can be expanded into Taylor series. The expansions of the width $b$ and depth $d$ in the element are:

$$
\begin{aligned}
& b=\sum_{n=0}^{h b} b_{i} c_{b n}(\Delta b)^{n}(s / l)^{n}=\mathbf{C}_{\mathbf{b}}\left\{\begin{array}{lllll}
1 & s / l & \ldots & (s / l)^{n} \ldots(s / l)^{h b}
\end{array}\right\}^{\mathrm{T}} \\
& d=\sum_{n=0}^{h b} d_{i} c_{d n}(\Delta d)^{n}(s / l)^{n}=\mathbf{C}_{\mathbf{d}}\left\{\begin{array}{lllll}
1 & s / l & \ldots & (s / l)^{n} \ldots(s / l)^{h d}
\end{array}\right\}^{\mathrm{T}}
\end{aligned}
$$

Where $h b$ and $h d$ are the truncation orders of width $b$ and depth $d$ respectively. The coefficient vectors are:

$$
\begin{aligned}
& \mathbf{C}_{\mathbf{b}}=\left[\begin{array}{lllll}
b_{i} c_{b 1} & b_{i} c_{b 2}(\Delta b) & \ldots & b_{i} c_{n}(\Delta b)^{n} \ldots b_{i} c_{b h b}(\Delta b)^{h b}
\end{array}\right] \\
& \mathbf{C}_{\mathbf{d}}=\left[\begin{array}{lllll}
d_{i} c_{d 1} & d_{i} c_{d 2}(\Delta d) & \ldots & d_{i} c_{n}(\Delta d)^{n} \ldots d_{i} c_{d h d}(\Delta d)^{h d}
\end{array}\right]
\end{aligned}
$$

Here $c_{b n}=t b(t b-1) \ldots(t b-n+1)(n !)^{-1}, c_{d n}=t d(t d-1) \ldots(t d-n+1)(n !)^{-1}$.

The power series of area and second moment inertia of cross section in the element can be obtained.

$$
\begin{aligned}
& A(x)=b d=\sum_{n=0}^{m_{A}} C_{A n}(s / l)^{n}=\mathbf{C}_{\mathbf{A}}\left\{\begin{array}{lllll}
1 & s / l & \ldots & (s / l)^{n} \ldots(s / l)^{m_{A}}
\end{array}\right\}^{\mathrm{T}} \\
& I(x)=\frac{b d^{3}}{12}=\sum_{n=0}^{m_{I}} C_{I n}(s / l)^{n}=\mathbf{C}_{\mathbf{I}}\left\{\begin{array}{llllll}
1 & s / l & \ldots & (s / l)^{n} \ldots(s / l)^{m_{I}}
\end{array}\right\}^{\mathrm{T}}
\end{aligned}
$$

Where the coefficient vector $\mathbf{C}_{\mathbf{A}}$ is the convolution of $\mathbf{C}_{\mathbf{b}}$ and $\mathbf{C}_{\mathbf{d}}$, and $\mathbf{C}_{\mathbf{I}}$ is the convolution of $\mathbf{C}_{\mathbf{b}}$ and $\left(\mathbf{C}_{\mathbf{d}}\right)^{3}$. $m_{A}$ and $m_{I}$ are the highest orders of area and second moment inertia of cross section respectively. For a beam with integer tapered order, $m_{A}=t b+t d$ and $m_{I}=t b+3 t d$. While for a beam with fraction tapered order, $m_{A}=h b+h d$ and $m_{I}=h b+3 h d$.

The mass and stiffness matrices for a tapered beam element can be obtained according to Eqs (20)-(23).

$$
\mathbf{M}_{t}=\rho \sum_{n=0}^{m_{A}} \mathbf{C}_{\mathbf{A}}(n) \mathbf{M}_{t n}
$$


Table 1

The Young's modulus of the materials of compressor and turbine under some temperatures [31]

\begin{tabular}{lccccccc}
\hline Temperature $\left({ }^{\circ} \mathrm{C}\right)$ & 24 & 100 & 200 & 300 & 400 & 500 & 600 \\
\hline Young's modulus of compressor material $\left(\times 10^{11} \mathrm{~Pa}\right)$ & 2.04 & 2.01 & 1.96 & 1.90 & 1.82 & 1.73 & 1.61 \\
Young's modulus of turbine material $\left(\times 10^{11} \mathrm{~Pa}\right)$ & 2.14 & 2.12 & 2.05 & 1.99 & 1.90 & 1.78 & 1.63 \\
\hline
\end{tabular}

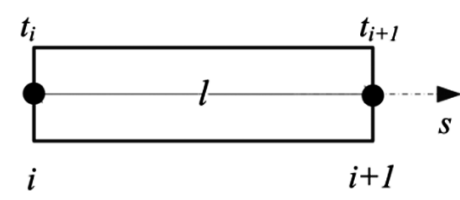

(a)

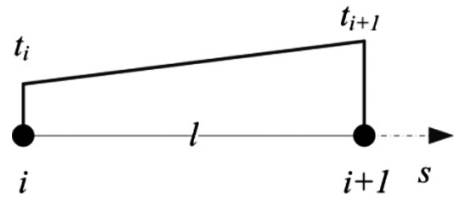

(b)

Fig. 5. A beam element with axial temperature distribution: (a) An element with nodal temperatures; (b) The temperature distribution along the axial direction.

$$
\begin{aligned}
& \mathbf{M}_{r}=\rho \sum_{n=0}^{m_{I}} \mathbf{C}_{\mathbf{I}}(n) \mathbf{M}_{r n} \\
& \mathbf{K}_{b}=E \sum_{n=0}^{m_{I}} \mathbf{C}_{\mathbf{I}}(n) \mathbf{K}_{b n} \\
& \mathbf{K}_{s}=\frac{12 E}{\phi l^{2}} \sum_{n=0}^{m_{I}} \mathbf{C}_{\mathbf{I}}(n) \mathbf{K}_{s n}
\end{aligned}
$$

According to Eqs (38)-(41), various tapered shapes can be considered by the improved beam element. Note that for the beams with fraction tapered orders, the truncation order $h b$ and $h d$ should be configured at first, which would have influence on the accuracy of the method.

With the beam element considering the variable axial parameters, the tapered beam can be modeled and analyzed by finite element method.

\subsection{Beams with non-uniform axial temperature distribution}

According to the Young's moduli under different temperatures in reference [31], as listed in Table 1, and the variation of Young's modulus with temperature in reference [30], the variation of Young's modulus with temperature can be written as:

$$
E=E_{0}\left(\gamma t^{2}+\beta t+\alpha\right)
$$

Where $E_{0}$ is the reference Young's modulus under reference temperature. $\gamma, \beta$ and $\alpha$ are the coefficients related to the particular material.

Some assumptions are employed in formulating the element with axial temperature distribution. The temperature of the cross section is assumed to be uniform and the expansion of the element due to temperature is neglected.

Assuming the temperature varies linearly along the axial direction, as shown in Fig. 5. The temperature in the element can be written as:

$$
t=t_{i}+\left(t_{i+1}-t_{i}\right)(s / l)=t_{i}+\Delta t(s / l)
$$

Taking Eq. (43) into Eq. (42), the variation of Young's modulus in the element can be obtained:

$$
E(s)=E_{0} f(s)
$$


Table 2

First four natral fequencies of a wedge beam with constant width and linearly tapered depth

\begin{tabular}{llcccc}
\hline Euler-Bernoulli beam & $\Omega_{1}$ & $\Omega_{2}$ & $\Omega_{3}$ & $\Omega_{4}$ \\
\hline Free-free & Reference [3] & 12.757509 & 27.755353 & 47.575945 & 72.294973 \\
& 1 element & 13.839018 & 85.839860 & - & - \\
& 2 elements & 12.911612 & 29.650459 & 72.829924 & 226.573769 \\
& 5 elements & 12.762865 & 27.893851 & 48.478618 & 75.810952 \\
& 10 element & 12.757858 & 27.766062 & 47.681793 & 72.820881 \\
& 20 element & 12.757531 & 27.756058 & 47.583657 & 72.342383 \\
& Reference [3] & 5.315099 & 15.207168 & 30.019809 & 49.763345 \\
& 1 element & 5.318737 & 17.300608 & - & - \\
& 2 elements & 5.315852 & 15.444351 & 32.702900 & 78.570387 \\
& 5 elements & 5.315123 & 15.216963 & 30.197645 & 50.760254 \\
& 10 elements & 5.315101 & 15.207821 & 30.034119 & 49.886813 \\
& 20 elements & 5.315100 & 15.207209 & 30.020759 & 49.772483 \\
\hline
\end{tabular}

Where $f(s)$ depends on the nodal temperature and the coordinate in the element. It can be written in terms of power series:

$$
f(s)=\gamma(\Delta t)^{2}(s / l)^{2}+\left(2 \gamma t_{i}+\beta\right)(\Delta t)(s / l)+\alpha+\beta t_{i}+\gamma t_{i}^{2}=\sum_{n=0}^{2} c_{n}(s / l)^{n}
$$

Where $c_{0}=\gamma t_{i}^{2}+\beta t_{i}+\alpha, c_{1}=2 \gamma t_{i}(\Delta t)+\beta(\Delta t)$ and $c_{2}=\gamma(\Delta t)^{2}$. The variation of Young's modulus has influence on the stiffness matrices of beam element, which can be written as:

$$
\begin{aligned}
& \mathbf{K}_{b}=\int_{0}^{l} E(s) I_{z}\left(\mathrm{~d} \mathbf{N}_{2} / \mathrm{d} s\right)^{\mathrm{T}}\left(\mathrm{d} \mathbf{N}_{2} / \mathrm{d} s\right) \mathrm{d} s=E_{0} I_{z} \sum_{n=0}^{2} c_{n} \mathbf{K}_{b n} \\
& \mathbf{K}_{s}=\int_{0}^{l} \frac{12 E(s) I_{z}}{\phi l^{2}} \mathbf{N}_{3}^{\mathrm{T}} \mathbf{N}_{\mathbf{3}} \mathrm{d} s=\frac{12 E_{0} I_{z}}{\phi l^{2}} \sum_{n=0}^{2} c_{n} \mathbf{K}_{s n}
\end{aligned}
$$

Since the mass matrices of beam element are not affected by the axial temperature distribution, they are identical to the traditional beam element.

With Eqs (46) and (47), the beams with linearly axial temperature distribution can be modeled and analyzed by the improved beam element. Note that the temperature distribution in the element can be of higher order variations, while more nodes of temperatures are required in the element.

\section{Numerical studies}

Beams with variable axial parameters can be modeled by the improved beam element. The mass and stiffness matrices can be obtained, and the governing equation of the beam is written as:

$$
\left(\mathbf{K}-\omega^{2} \mathbf{M}\right)\{\mathbf{X}\}=\{\mathbf{Q}\}
$$

Where $\mathbf{M}$ and $\mathbf{K}$ are the mass and stiffness matrices of the beam, $\omega$ is the vibration frequency. $\{\mathbf{X}\}$ is the displacement vector of vibration, $\{\mathbf{Q}\}$ is the load vector. The natural frequencies and modal shapes of the beam can be obtained. The dimensionless natural frequency is defined as:

$$
\Omega=\omega L^{2} \sqrt{\rho A /\left(E I_{z}\right)}
$$

Where $L$ is the length of the beam, $A$ and $I_{z}$ are the area and second moment inertia of cross section respectively. For tapered beams, $A$ and $I_{z}$ are the area and second moment inertia of the cross section at the large end. $\rho$ is the density, $E$ is the Young's modulus. For beams under non-uniform temperature distributions, the value of $E$ under $20^{\circ} \mathrm{C}$ is applied in Eq. (49).

With the improved beam element, tapered beams with various tapered orders are modeled and the transverse vibration will be studied. Moreover, a uniform beam under linearly axial temperature distribution is to be investigated. 
Table 3

First three natural fequencies of a wedge beam with parabolic tapered width and depth

\begin{tabular}{|c|c|c|c|c|}
\hline \multicolumn{2}{|c|}{ Euler-Bernoulli beam } & \multirow{2}{*}{$\frac{\Omega_{1}}{20.042}$} & \multirow{2}{*}{$\frac{\Omega_{2}}{47.921}$} & \multirow{2}{*}{$\frac{\Omega_{3}}{86.870}$} \\
\hline Free-free & Reference [6] & & & \\
\hline & 1 element & 20.3166 & 92.4513 & \\
\hline & 2 elements & 20.1996 & 49.1519 & 107.1107 \\
\hline & 5 elements & 20.0474 & 48.0449 & 87.6717 \\
\hline & 10 elements & 20.0466 & 47.9329 & 86.9184 \\
\hline & 20 elements & 20.0467 & 47.9310 & 86.8639 \\
\hline \multirow[t]{6}{*}{ Free-clamped } & Reference [6] & 6.2997 & 22.724 & 50.481 \\
\hline & 1 element & 6.3117 & 23.0513 & \\
\hline & 2 elements & 6.2948 & 22.8843 & 51.8033 \\
\hline & 5 elements & 6.2989 & 22.7211 & 50.5940 \\
\hline & 10 elements & 6.2989 & 22.7198 & 50.5732 \\
\hline & 20 elements & 6.2987 & 22.7203 & 50.4711 \\
\hline
\end{tabular}

Table 4

First four dimensionless natural fequencies of a cantilever Timoshenko beam

\begin{tabular}{|c|c|c|c|c|c|}
\hline \multicolumn{2}{|c|}{ Timoshenko beam } & $\Omega_{1}$ & $\Omega_{2}$ & $\Omega_{3}$ & $\Omega_{4}$ \\
\hline \multirow[t]{6}{*}{ Free-clamped } & Reference [7] & 6.4098 & 21.145 & 40.929 & 61.385 \\
\hline & 1 element & 6.4834 & 28.8975 & & \\
\hline & 2 elements & 6.4692 & 22.1154 & 62.1312 & 88.0347 \\
\hline & 5 elements & 6.4327 & 21.4653 & 43.0167 & 67.7383 \\
\hline & 10 elements & 6.4229 & 21.2931 & 41.6328 & 63.4297 \\
\hline & 20 elements & 6.4201 & 21.2466 & 41.2659 & 62.1859 \\
\hline
\end{tabular}
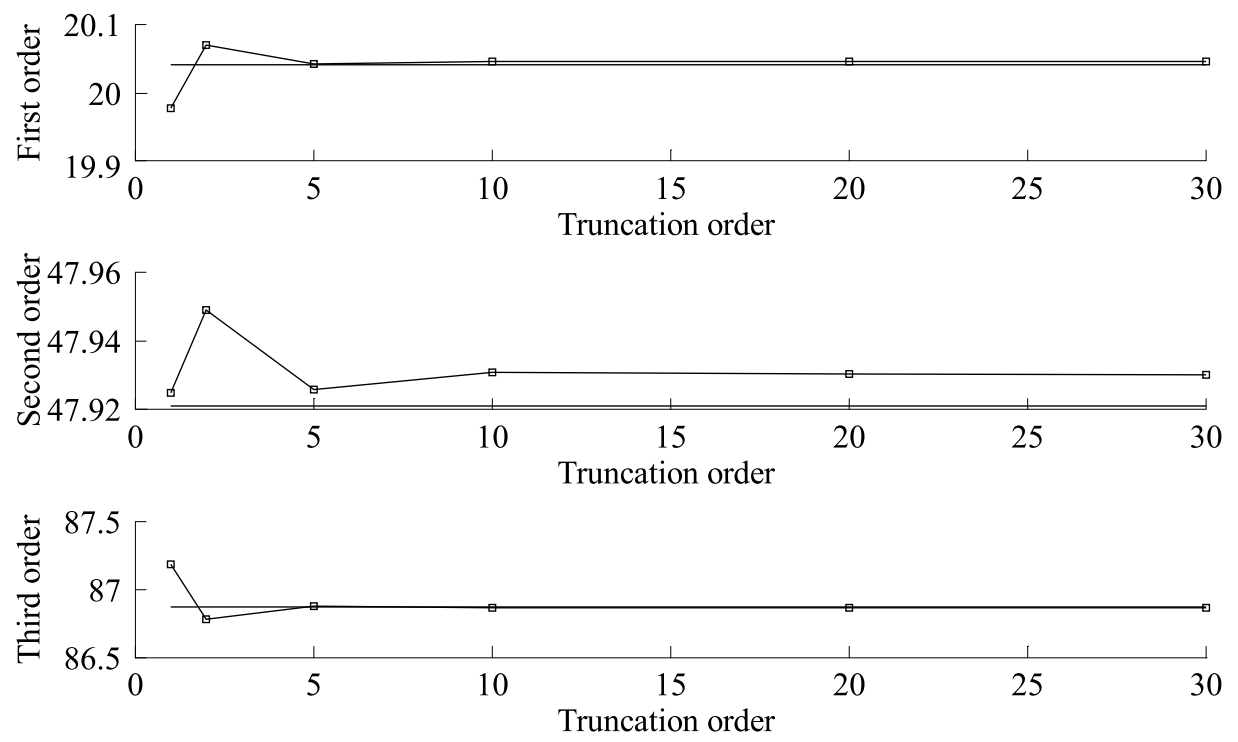

“__ relates to the theoretical natural frequencies in [6].

“世” indicates the results of this work.

Fig. 6. The variations of first three natural frequencies with the truncation orders.

\subsection{Tapered beams}

The improved beam element is verified by comparing with some published results. A wedge beam with constant width and linearly tapered depth is studied and compared with reference [3]. The natural frequencies are listed in Table 2 . It can be seen that the improved beam element predicts the natural frequencies accurately with few elements. 


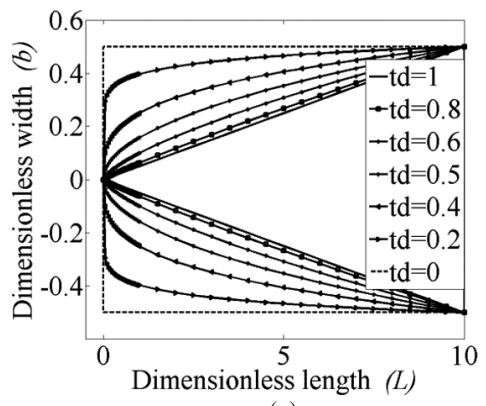

(a)

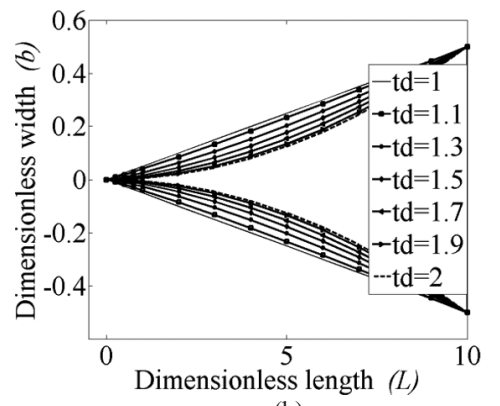

(b)

Fig. 7. The shapes of beams with various tapered orders: (a) tapered order varies from 0 to 1 with step 0.1 , (b) tapered order varies from 1 to 2 with step 0.1 .

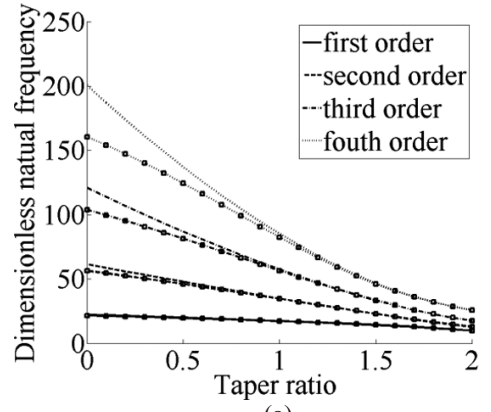

(a)

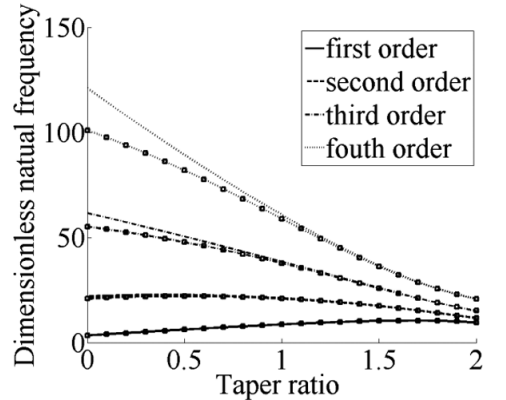

(b)

Fig. 8. The variation of first four natural frequencies with tapered orders of a wedge beam: (a) free-free boundary condition, (b) free-clamped boundary condition. The line with " $\square$ " are the results of Timoshenko beam, without " $\square$ " are the results of Euler-Bernoulli beam.

An Euler-Bernoulli beam with parabolic tapered width and depth is studied and compared with reference [6]. The truncation orders of Taylor expansions should be configured for the beam with fraction tapered orders. In order to reduce the influence of element number, the beam is modeled by 20 elements. Figure 6 presents the variations of the first three natural frequencies with truncation order. It can be seen that the natural frequencies are almost not affected when the truncation order is larger than 10. As a result, the truncation order will be set as 10 in the following study of the beams with fraction tapered orders.

The transverse vibration of a double parabolic tapered beam is studied with different number of elements. The comparison with reference [6] is listed in Table 3. The frequencies predicted by the finite element method with few elements are close to the analytical results.

A cantilever Timoshenko beam with constant depth and linearly tapered width is studied and compared with reference [7]. As shown in Table 4, the differences of the first four natural frequencies are less than $0.204 \%$ between reference [7] and the improved beam element (with 10 elements). The finite element model with 10 improved beam elements could predict the natural frequencies accurately.

With 10 elements and 10 expansions, a series of tapered beams with tapered order varying form 0 (uniform beam) to 2 are studied. The shapes of the beams with different tapered orders are shown in Fig. 7. With the improved beam element, the tapered beams are studied by Euler-Bernoulli beam theory and Timoshenko beam theory. The free-free and free-clamped boundary conditions are both considered in vibration analysis. In free-clamped case, the large end of taper beam is clamped. Figure 8 presents the first four natural frequencies of different tapered orders. It can be seen that the natural frequencies predicted by Timoshenko beam theory are less than Euler-Bernoulli theory. The differences of the two beam theories decrease with the increase of the taper order. This is due to the increase of tapered order makes the beam become more and more slender, especially at the sharp end of the tapered beam. The contribution of rotary inertia and the shear effect have little influence on the transverse vibration of slender beams. As a result, the natural frequencies predicted by the two beam theories are close for higher taper orders. It can be 


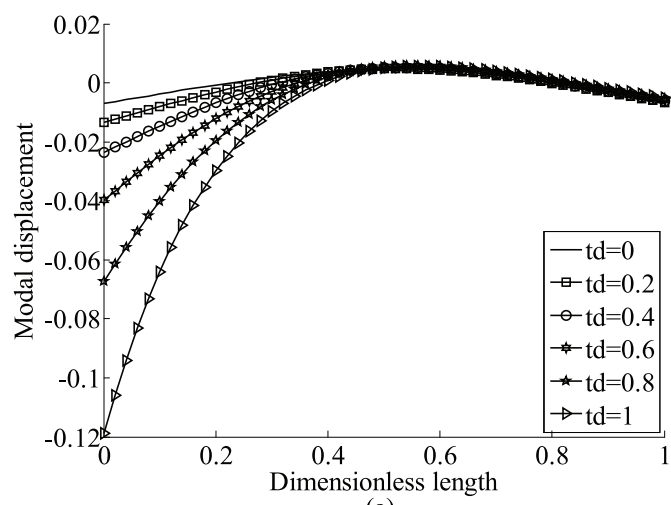

(a)

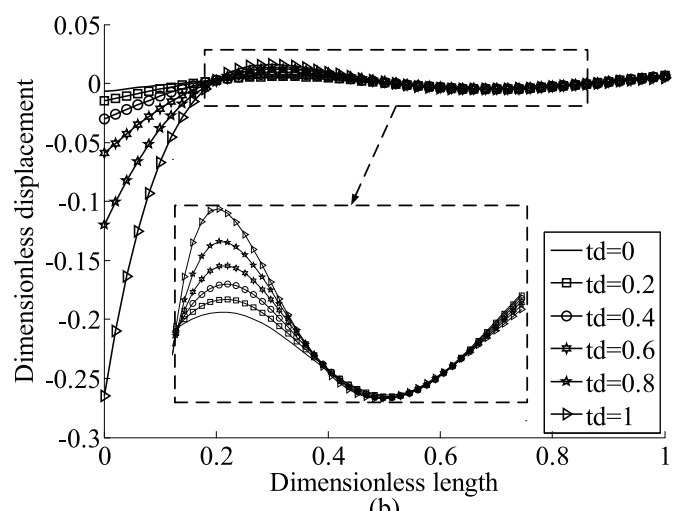

(b)

Fig. 9. The variation of first two modal shapes with tapered orders of beams under free-free boundary condition: (a) the first modal shapes, (b) the second modal shapes.

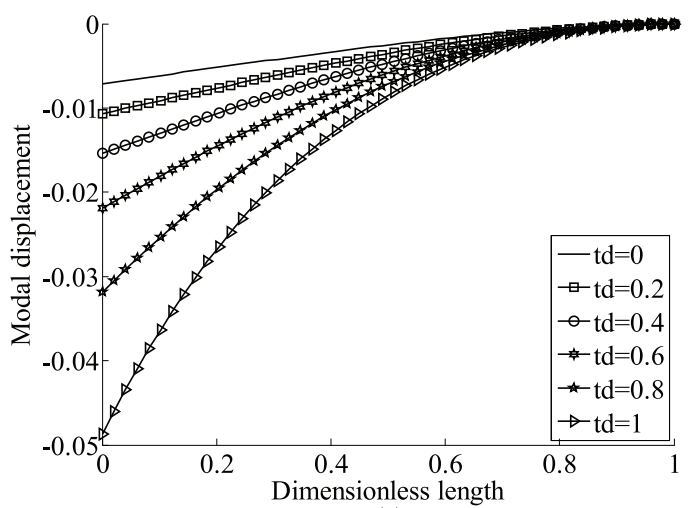

(a)

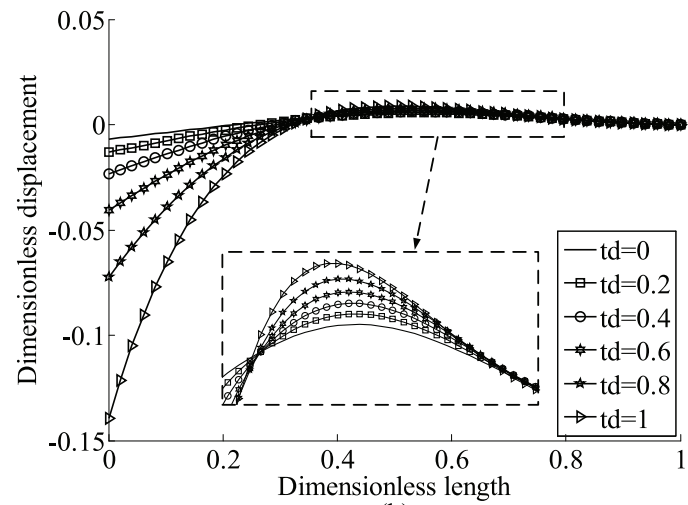

(b)

Fig. 10. The variation of first two modal shapes with tapered orders of beams under free-clamped boundary condition: (a) the first modal shapes, (b) the second modal shapes.

concluded that the differences of the natural frequencies between the two beam theories can be neglected when the tapered order is larger than 2 .

The modal shapes of several tapered orders under free-free and free-clamped conditions are given in Figs 9 and 10 respectively. With the increase of tapered order, the modal displacement of the free sharp end increases dramatically. The vibration of the tapered beam will be mainly happened at the free sharp end for high tapered orders.

Note that Zhou and Cheung [6,7] investigated the free vibration of this type of tapered beams analytically. With expanding the load into Taylor series, they derived a static beam function and applied the Rayleigh-Ritz method to study the vibration of the beam with this taper shape. Different from their work, this study expands the variable axial parameters in terms of power series and modeled the tapered beam by finite element method.

The vibration analysis of various tapered beam indicates that the improved beam element is reliable and convenient to consider the various taper shape of beam.

\subsection{Beams with axial temperature distribution}

The beams with non-uniform axial temperature distribution can be modeled by the improved beam element. A beam with linearly axial temperature distribution will be studied by the improved element derived in Section 2.3.

In order to validate the beam model, a uniform beam under linearly axial temperature distribution is studied. The reference Young's modulus $E_{0}$ is set as $200 \mathrm{GPa}$, and the coefficients are $\gamma=-2.978 E-7, \beta=-1.873 E-4$, $\alpha=1.002$. The temperature of the cold end is $20^{\circ} \mathrm{C}$, and the axial temperature difference is set as $100^{\circ} \mathrm{C}$. The 


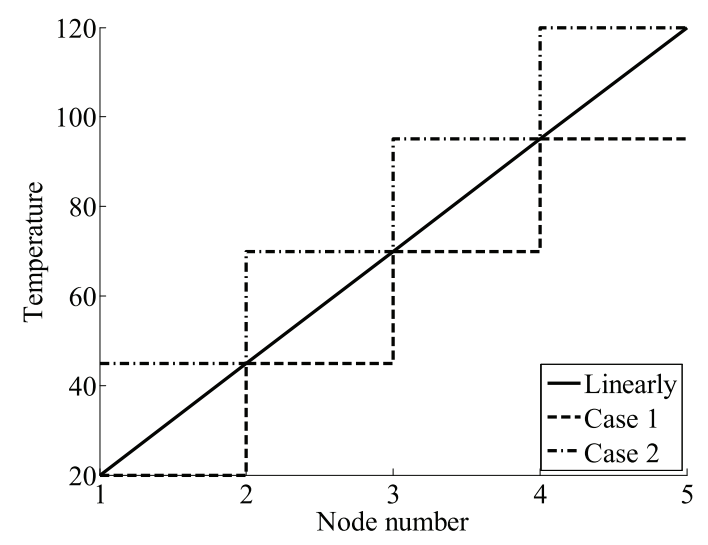

Fig. 11. The three treatments of axial temperature distribution.

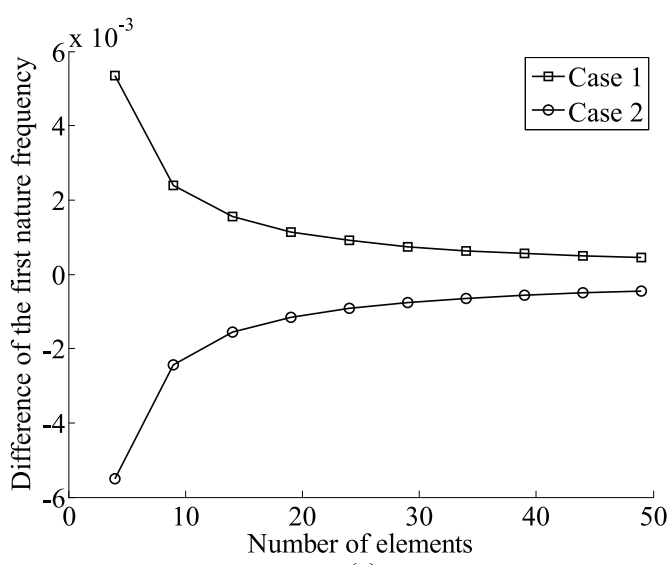

(a)

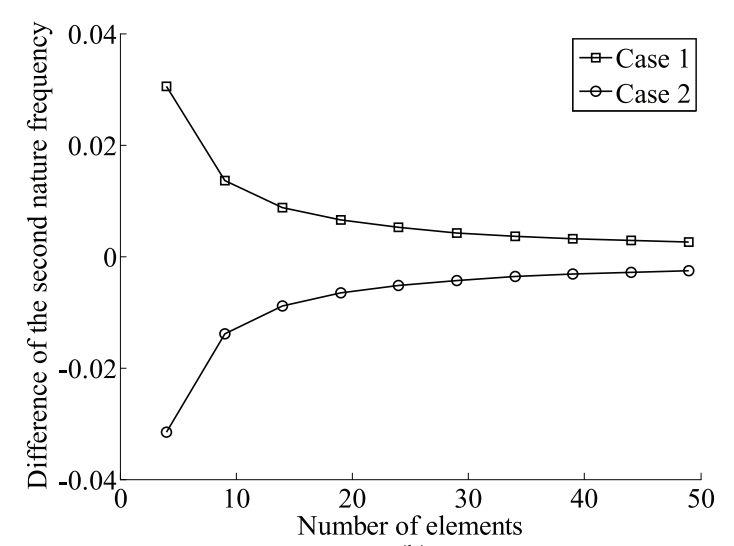

(b)

Fig. 12. The differences of the first two nature frequencies of the traditional beam element form the improved beam element. (a) The differences of the first nature frequencies; (b) The differences of the second nature frequencies.

beam can be modeled by the improved beam element which considers the linearly axial temperature distribution in the element, or by the traditional beam element with constant temperature in each element. Assume the beam is modeled by 4 beam elements, Fig. 11 shows the three treatments of the temperature distribution in beam element. The linearly temperature distribution can be directly considered by the improved beam element, while for traditional beam element the constant temperature in the beam can be selected as the nodal temperatures. As given in Fig. 11, the lower nodal temperature is selected in Case 1, and the higher nodal temperature is selected in Case 2.

The transverse vibration of the beam under linearly axial temperature distribution is studied by the improved beam element and the traditional beam element. The differences of the natural frequencies predicted by traditional beam element and the improved beam element are shown in Fig. 12. Since the temperature of each element in Case 1 is less than linearly distribution, Case 1 predicts greater nature frequencies than this work. Accordingly Case 2 predicts less natural frequencies than this work. With the increase of element number, the two cases gradually approach this work, which validates the beam model under linearly axial temperature distribution in this work.

A uniform beam with linearly axial temperature distribution is studied under free-free and free-clamped boundary conditions respectively. The variations of the first three natural frequencies with the axial temperature difference $D_{t}$ are plotted in Fig. 13. With the axial temperature difference increasing to $1000^{\circ} \mathrm{C}$, the natural frequencies decrease about $11 \%$ in free-free condition, while the first three natural frequencies of a free-clamped beam decrease about $22 \%, 15 \%$ and $13 \%$ respectively.

The influence of axial temperature difference on the modal shapes of beam can be investigated. As shown in Fig. 14, the axial temperature distribution has little influence on the first modal shape, while the influence on the 


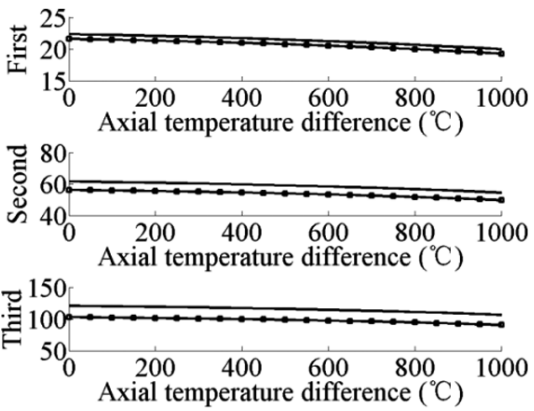

(a)
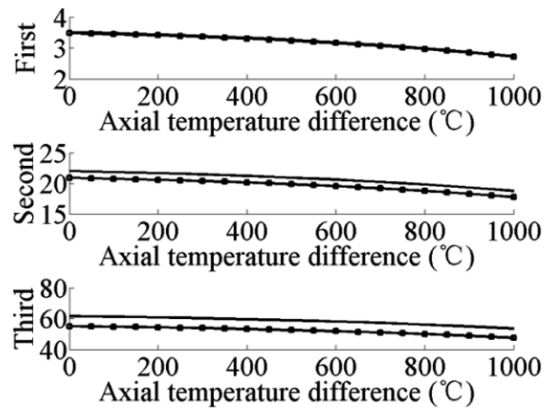

(b)

Fig. 13. Variation of the first three dimensionless natural frequencies with the maximum axial temperature difference of a beam under linearly varying axial temperature distribution: (a) free-free boundary condition, (b) free-clamped boundary condition. "Unmarked" curves relate to results of Euler-Bernoulli beam, "marked" curves relate to the results of Timoshenko beam.

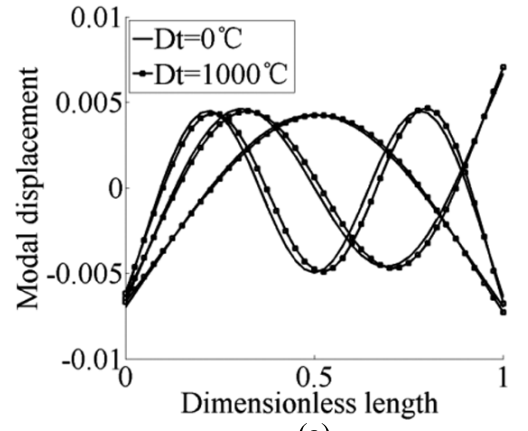

(a)

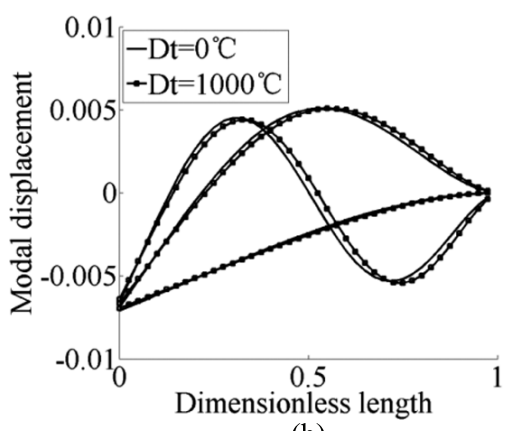

(b)

Fig. 14. The comparison of first three modal shapes under 0 axial temperature difference and $1000^{\circ}$ axial temperature difference: (a) free-free boundary condition, (b) free-clamped boundary condition.

second and third modal shapes is obvious.

It is note that the beams under various temperature distributions can be modeled with improved beam element. Although only linearly temperature distribution is studied here, it still can be of higher order temperature distributions, while more nodes of temperature are required.

With the study of various tapered beams and a beam under linearly axial temperature distribution, it can be found that the improved beam element can consider the variable axial parameters of beam conveniently.

\section{Conclusions}

The beam element is improved to consider the variable axial parameters. The variations of axial parameters are formulated in terms of a power series, and the elementary matrices which depend on the power orders are derived. The matrices of the improved beam element can be obtained by a matrix series with the elementary matrices. The beams with various tapered shapes and a beam under linearly axial temperature distribution are studied by the improved beam element.

Various tapered beams are studied by finite element method with the improved beam element. Comparisons with some published results validate the improved beam element. The variations of natural frequencies and modal shapes with tapered orders are investigated. A uniform beam with linearly axial temperature distribution is modeled by the improved beam element. The influences of axial temperature distribution on the natural frequencies and modal shapes are investigated.

The cases studied indicate that the improved beam element is flexible and reliable to consider the variable axial parameters of beams. The transverse vibration of beams with various tapered orders or under non-uniform axial temperature distribution can be studied conveniently by the improved beam element. 


\section{Acknowledgment}

The authors thank for the support of the 863 project (2008AA05A302) grant of Ministry of Science and Technology, China.

\section{Appendix}

A1: The details of the shape functions $\mathbf{N}_{1}$ and $\mathbf{N}_{2}$ are list as follows:

$$
\begin{aligned}
& N_{11}=\frac{1}{1+\phi}\left[1+\phi\left(1-\left(\frac{s}{l}\right)\right)-3\left(\frac{s}{l}\right)^{2}+2\left(\frac{s}{l}\right)^{3}\right] \\
& N_{12}=\frac{l}{1+\phi}\left(\frac{s}{l}\right)\left[1+\frac{1}{2} \phi\left(1-\left(\frac{s}{l}\right)\right)-2\left(\frac{s}{l}\right)+\left(\frac{s}{l}\right)^{2}\right] \\
& N_{13}=\frac{1}{1+\phi}\left(\frac{s}{l}\right)\left[3\left(\frac{s}{l}\right)-2\left(\frac{s}{l}\right)^{2}+\phi\right] \\
& N_{14}=\frac{l}{1+\phi}\left(\frac{s}{l}\right)\left[-\frac{1}{2} \phi\left(1-\left(\frac{s}{l}\right)\right)-\left(\frac{s}{l}\right)+\left(\frac{s}{l}\right)^{2}\right] \\
& N_{21}=\frac{1}{l(1+\phi)}\left[6\left(\frac{s}{l}\right)\left(\left(\frac{s}{l}\right)-1\right)\right] \\
& N_{22}=\frac{1}{1+\phi}\left[1-4\left(\frac{s}{l}\right)+3\left(\frac{s}{l}\right)^{2}+\phi\left(1-\left(\frac{s}{l}\right)\right)\right] \\
& N_{23}=-\frac{6}{l(1+\phi)}\left(\frac{s}{l}\right)\left(\left(\frac{s}{l}\right)-1\right) \\
& N_{24}=\frac{1}{1+\phi}\left[-2\left(\frac{s}{l}\right)+3\left(\frac{s}{l}\right)^{2}+\phi\left(\frac{s}{l}\right)\right]
\end{aligned}
$$

A2: General forms of matrices of $\mathbf{M}_{t n}, \mathbf{M}_{r n}, \mathbf{K}_{b n}$ and $\mathbf{K}_{s n}$.

$$
\mathbf{M}_{t n}=\int_{0}^{l}\left(\frac{s}{l}\right)^{n} \mathbf{N}_{1}^{\mathrm{T}} \mathbf{N}_{1} \mathrm{~d} s=\left[\begin{array}{llll}
m_{t 11} & m_{t 12} & m_{t 13} & m_{t 14} \\
& m_{t 22} & m_{t 23} & m_{t 24} \\
& & m_{t 33} & m_{t 34} \\
& & & m_{t 44}
\end{array}\right]
$$

Where $m_{t i j}=\int_{0}^{l}(s / l)^{n} N_{1 i} N_{1 j} d s$. The elements of $\mathbf{M}_{b n}$ can be derived:

$$
\begin{aligned}
& m_{t 11}=\frac{l}{(1+\phi)^{2}}\left[\frac{1+2 \phi+\phi^{2}}{n+1}-\frac{2 \phi+2 \phi^{2}}{n+2}+\frac{-6-6 \phi+\phi^{2}}{n+3}+\frac{4+10 \phi}{n+4}+\frac{9-4 \phi}{n+5}-\frac{12}{n+6}+\frac{4}{n+7}\right] \\
& m_{t 12}=\frac{l^{2}}{(1+\phi)^{2}}\left[\frac{1+\frac{3}{2} \phi+\frac{1}{2} \phi^{2}}{n+2}-\frac{2+\frac{7}{2} \phi+\phi^{2}}{n+3}+\frac{-2+\frac{3}{2} \phi+\frac{1}{2} \phi^{2}}{n+4}+\frac{8+\frac{3}{2} \phi}{n+5}-\frac{7+\phi}{n+6}+\frac{2}{n+7}\right] \\
& m_{t 13}=\frac{l}{(1+\phi)^{2}}\left[\frac{\phi+\phi^{2}}{n+2}+\frac{3+3 \phi-\phi^{2}}{n+3}-\frac{2+8 \phi}{n+4}+\frac{-9+4 \phi}{n+5}+\frac{12}{n+6}-\frac{4}{n+7}\right] \\
& m_{t 14}=\frac{l^{2}}{(1+\phi)^{2}}\left[\frac{-\frac{1}{2} \phi-\frac{1}{2} \phi^{2}}{n+2}+\frac{-1-\frac{1}{2} \phi+\phi^{2}}{n+3}+\frac{1+\frac{7}{2} \phi-\frac{1}{2} \phi^{2}}{n+4}+\frac{3-\frac{7}{2} \phi}{n+5}+\frac{-5+\phi}{n+6}+\frac{2}{n+7}\right] \\
& m_{t 22}=\frac{l^{3}}{(1+\phi)^{2}}\left[\frac{1+\phi+\frac{1}{4} \phi^{2}}{n+3}-\frac{4+3 \phi+\frac{1}{2} \phi^{2}}{n+4}+\frac{6+3 \phi+\frac{1}{2} \phi^{2}}{n+5}-\frac{4+\phi}{n+6}+\frac{1}{n+7}\right]
\end{aligned}
$$




$$
\begin{aligned}
& m_{t 23}=\frac{l^{2}}{(1+\phi)^{2}}\left[\frac{\phi+\frac{1}{2} \phi^{2}}{n+3}+\frac{3-\frac{1}{2} \phi-\frac{1}{2} \phi^{2}}{n+4}-\frac{8+\frac{3}{2} \phi}{n+5}+\frac{7+\phi}{n+6}-\frac{2}{n+7}\right] \\
& m_{t 24}=\frac{l^{3}}{(1+\phi)^{2}}\left[\frac{-\frac{1}{2} \phi-\frac{1}{4} \phi^{2}}{n+3}+\frac{-1+\phi+\frac{1}{2} \phi^{2}}{n+4}+\frac{3-\frac{1}{2} \phi-\frac{1}{4} \phi^{2}}{n+5}-\frac{3}{n+6}+\frac{1}{n+7}\right] \\
& m_{t 33}=\frac{l}{(1+\phi)^{2}}\left[\frac{\phi^{2}}{n+3}+\frac{6 \phi}{n+4}+\frac{9-4 \phi}{n+5}-\frac{12}{n+6}+\frac{4}{n+7}\right] \\
& m_{t 34}=\frac{l^{2}}{(1+\phi)^{2}}\left[\frac{-\frac{1}{2} \phi^{2}}{n+3}+\frac{-\frac{5}{2} \phi+\frac{1}{2} \phi^{2}}{n+4}+\frac{-3+\frac{7}{2} \phi}{n+5}+\frac{5-\phi}{n+6}-\frac{2}{n+7}\right] \\
& m_{t 44}=\frac{l^{3}}{(1+\phi)^{2}}\left[\frac{\frac{1}{4} \phi^{2}}{n+3}+\frac{\phi-\frac{1}{2} \phi^{2}}{n+4}+\frac{1-2 \phi+\frac{1}{4} \phi^{2}}{n+5}+\frac{-2+\phi}{n+6}+\frac{1}{n+7}\right] \\
& \mathbf{M}_{r n}=\int_{0}^{l}\left(\frac{s}{l}\right)^{n} \mathbf{N}_{2}^{\mathrm{T}} \mathbf{N}_{2} \mathrm{~d} s=\left[\begin{array}{llll}
m_{r 11} & m_{r 12} & m_{r 13} & m_{r 14} \\
& m_{r 22} & m_{r 23} & m_{r 24} \\
& & m_{r 33} & m_{r 34} \\
& & & m_{r 44}
\end{array}\right]
\end{aligned}
$$

Where $m_{r i j}=\int_{0}^{l}(s / l)^{n} N_{2 i} N_{2 j} d s$. The elements of $\mathbf{M}_{r n}$ can be derived:

$$
\begin{aligned}
& m_{r 11}=\frac{1}{l(1+\phi)^{2}}\left[\frac{36}{n+3}-\frac{72}{n+4}+\frac{36}{n+5}\right] \\
& m_{r 12}=\frac{1}{(1+\phi)^{2}}\left[\frac{-6-6 \phi}{n+2}+\frac{30+12 \phi}{n+3}-\frac{42+6 \phi}{n+4}+\frac{18}{n+5}\right] \\
& m_{r 13}=\frac{1}{l(1+\phi)^{2}}\left[\frac{-36}{n+3}+\frac{72}{n+4}-\frac{36}{n+5}\right] \\
& m_{r 14}=\frac{1}{(1+\phi)^{2}}\left[\frac{12-6 \phi}{n+3}+\frac{-30+6 \phi}{n+4}+\frac{18}{n+5}\right] \\
& m_{r 22}=\frac{l}{(1+\phi)^{2}}\left[\frac{1+2 \phi+\phi^{2}}{n+1}-\frac{8+10 \phi+2 \phi^{2}}{n+2}+\frac{22+14 \phi+\phi^{2}}{n+3}-\frac{24+6 \phi}{n+4}+\frac{9}{n+5}\right] \\
& m_{r 23}=\frac{1}{(1+\phi)^{2}}\left[\frac{6+6 \phi}{n+2}-\frac{30+12 \phi}{n+3}+\frac{42+6 \phi}{n+4}-\frac{18}{n+5}\right] \\
& m_{r 24}=\frac{l}{(1+\phi)^{2}}\left[\frac{-2-\phi+\phi^{2}}{n+2}+\frac{11+\phi-\phi^{2}}{n+3}-\frac{18}{n+4}+\frac{9}{n+5}\right] \\
& m_{r 33}=\frac{1}{l(1+\phi)^{2}}\left[\frac{36}{n+3}-\frac{72}{n+4}+\frac{36}{n+5}\right] \\
& m_{r 14}=\frac{1}{(1+\phi)^{2}}\left[\frac{6 \phi-12}{n+3}+\frac{30-6 \phi}{n+4}-\frac{18}{n+5}\right] \\
& m_{r 44}=\frac{l}{(1+\phi)^{2}}\left[\frac{4-\phi+\phi^{2}}{n+3}-\frac{12-6 \phi}{n+4}+\frac{9}{n+5}\right] \\
& \mathbf{K}_{b n}=\int_{0}^{l}\left(\frac{s}{l}\right)\left(\frac{\mathrm{d} \mathbf{N}_{2}}{\mathrm{~d} s}\right)^{\mathrm{T}}\left(\frac{\mathrm{d} \mathbf{N}_{2}}{\mathrm{~d} s}\right) \mathrm{d} s=\left[\begin{array}{llll}
k_{b 11} & k_{b 12} & k_{b 13} & k_{b 14} \\
& k_{b 22} & k_{b 23} & k_{b 24} \\
& & k_{b 33} & k_{b 34} \\
& & & k_{b 44}
\end{array}\right]
\end{aligned}
$$


Where $k_{b n}=\int_{0}^{l}(s / l)^{n}\left(d N_{2 i} / d s\right)\left(d N_{2 j} / d s\right) d s$. The elements of $\mathbf{K}_{b n}$ can be derived:

$$
\begin{aligned}
& k_{b 11}=\frac{1}{l^{3}(1+\phi)^{2}}\left[\frac{36}{n+1}-\frac{144}{n+2}+\frac{144}{n+3}\right] \\
& k_{b 12}=\frac{1}{l^{2}(1+\phi)^{2}}\left[\frac{24+6 \phi}{n+1}-\frac{84+12 \phi}{n+2}+\frac{72}{n+3}\right] \\
& k_{b 13}=\frac{1}{l^{3}(1+\phi)^{2}}\left[-\frac{36}{n+1}+\frac{144}{n+2}-\frac{144}{n+3}\right] \\
& k_{b 14}=\frac{1}{l^{2}(1+\phi)^{2}}\left[\frac{12-6 \phi}{n+1}-\frac{60-12 \phi}{n+2}+\frac{72}{n+3}\right] \\
& k_{b 22}=\frac{1}{l(1+\phi)^{2}}\left[\frac{16+8 \phi+\phi^{2}}{n+1}-\frac{48+12 \phi}{n+2}+\frac{36}{n+3}\right] \\
& k_{b 23}=\frac{1}{l^{2}(1+\phi)^{2}}\left[-\frac{24+6 \phi}{n+1}+\frac{84+12 \phi}{n+2}-\frac{72}{n+3}\right] \\
& k_{b 24}=\frac{1}{l(1+\phi)^{2}}\left[\frac{8-2 \phi-\phi^{2}}{n+1}-\frac{36}{n+2}+\frac{36}{n+3}\right] \\
& k_{b 33}=\frac{1}{l^{3}(1+\phi)^{2}}\left[\frac{36}{n+1}-\frac{144}{n+2}+\frac{144}{n+3}\right] \\
& k_{b 34}=\frac{1}{l^{2}(1+\phi)^{2}}\left[-\frac{12-6 \phi}{n+1}+\frac{60-12 \phi}{n+2}-\frac{72}{n+3}\right] \\
& k_{b 44}=\frac{1}{l(1+\phi)^{2}}\left[\frac{4-4 \phi+\phi^{2}}{n+1}-\frac{24-12 \phi}{n+2}+\frac{36}{n+3}\right] \\
& \mathbf{K}_{s n}=\int_{0}^{l}\left(\frac{s}{l}\right)^{n} \mathbf{N}_{3}^{\mathrm{T}} \mathbf{N}_{\mathbf{3}} \mathrm{d} s=\left[\begin{array}{llll}
k_{s 11} & k_{s 12} & k_{s 13} & k_{s 14} \\
& k_{s 22} & k_{s 23} & k_{s 24} \\
& & k_{s 33} & k_{s 34} \\
& & & k_{s 44}
\end{array}\right]
\end{aligned}
$$

Where $k_{s i j}=\int_{0}^{l}(s / l)^{n} N_{3 i} N_{3 j} d s$. The elements of $\mathbf{K}_{s n}$ can be derived:

$$
\begin{aligned}
& k_{s 11}=\frac{\phi^{2}}{l(1+\phi)^{2}} \frac{1}{n+1} ; \\
& k_{s 12}=\frac{\phi^{2}}{2(1+\phi)^{2}} \frac{1}{n+1} ; \\
& k_{s 22}=\frac{l \phi^{2}}{4(1+\phi)^{2}} \frac{1}{n+1} ; \\
& k_{s 13}=-k_{s 11} ; k_{s 14}=k_{s 12} ; k_{s 23}=-k_{s 12} ; k_{s 24}=k_{s 22} ; k_{s 33}=k_{s 11} ; k_{s 34}=-k_{s 12} ; k_{s 44}=k_{s 22}
\end{aligned}
$$

\section{References}

[1] S. Naguleswaran, Vibration of an Euler-Bernoulli beam of constant depth and with linearly varying breadth, J Sound Vib 153 (1992), 509-522.

[2] S. Naguleswaran, Vibration in the two principal planes of a non-uniform beam of rectangular cross-section, one side of which varies as the square root of the axial co-ordinate, J Sound Vib 172 (1994), 305-319. 
[3] S. Naguleswaran, A direct solution for the transverse vibration of Euler-Bernoulli wedge and cone beams, J Sound Vib 172 (1994), 289304.

[4] S. Abrate, Vibration of non-uniform rods and beams, J Sound Vib 185 (1995), 703-716.

[5] N.M. Auciello and A. Ercolano, Exact solution for the transverse vibration of a beam a part of which is a taper beam and other part is a uniform beam, Int J Solids Struct 34 (1997), 2115-2129.

[6] D. Zhou and Y.K. Cheung, The free vibration of a type of tapered beams, Comput Method Appl M 188 (2000), 203-216.

[7] D. Zhou and Y.K. Cheung, Vibrations of tapered Timoshenko beams in terms of static Timoshenko beam functions, Journal of Applied Mechanics 68 (2001), 596-602.

[8] J.S. Wu and D.W. Chen, Bending vibrations of wedge beams with any number of point masses, J Sound Vib 262 (2003), $1073-1090$.

[9] J.S. Wu and L.K. Chiang, Free vibrations of solid and hollow wedge beams with rectangular or circular cross-sections and carrying any number of point masses, Int J Numer Meth Eng 60 (2004), 695-718.

[10] M.C. Ece, M. Aydogdu and V. Taskin, Vibration of a variable cross-section beam, Mech Res Commun 34 (2007), 78-84.

[11] D.I. Caruntu, Classical Jacobi polynomials, closed-form solutions for transverse vibrations, J Sound Vib 306 (2007), $467-497$.

[12] D.I. Caruntu, Dynamic modal characteristics of transverse vibrations of cantilevers of parabolic thickness, Mech Res Commun 36 (2009), 391-404.

[13] R.D. Firouz-Abadi, H. Haddadpour and A.B. Novinzadeh, An asymptotic solution to transverse free vibrations of variable-section beams, J Sound Vib 304 (2007), 530-540.

[14] J.C. Hsu, H.Y. Lai and C.K. Chen, Free vibration of non-uniform Euler-Bernoulli beams with general elastically end constraints using Adomian modified decomposition method, J Sound Vib 318 (2008), 965-981.

[15] O.O. Ozdemir and M.O. Kaya, Vibration analysis of a rotating tapered Timoshenko beam using DTM, Meccanica 45 (2010), $33-42$.

[16] L. Klein, Transverse vibrations of non-uniform beams, J Sound Vib 37 (1974), 491-505.

[17] C.W.S. To, Higher order tapered beam finite elements for vibration analysis, J Sound Vib 63 (1979), 33-50.

[18] C.W.S. To, A linearly tapered beam finite element incorporating shear deformation and rotary inertia for vibration analysis, $J$ Sound Vib 78 (1981), 475-484.

[19] K.E. Rouch and J.S. Kao, A tapered beam finite element for rotor dynamics analysis, J Sound Vib 66 (1979), 119-140.

[20] L.M. Greenhill, W.B. Bickford and H.D. Nelson, A conical beam finite element for rotor dynamics analysis, Journal of Vibration, Acoustics, Stress and Reliability in Design 107 (1985), 421-430.

[21] G. Genta and A. Gugliotta, A conical element for finite element rotor dynamics, J Sound Vib 120 (1988), 175-182.

[22] W.L. Cleghorn and B. Tabarrok, Finite element formulation of a tapered Timoshenko beam for free lateral vibration analysis, $J$ Sound Vib 152 (1992), 461-470.

[23] G. Wang and N.M. Wereley, Free vibration analysis of rotating blades with uniform tapers, Aiaa J 42 (2004), $2429-2437$.

[24] R. Banerjee and J. Ewen, Dynamic stiffness formulation using timoshenko theory for free vibration of rotating beams, in: $48^{\text {th }}$ AIAA/ASME/ASCE/AHS/ASC Structures, Structural Dynamics and Materials Conference, Honolulu, Hawaii, 2007.

[25] G.J. Babu and R. Ganguli, New rational interpolation functions for finite element analysis of rotating beams, Int J Mech Sci $\mathbf{5 0}$ (2008), $578-588$.

[26] J.B. Gunda, R.K. Gupta and R. Ganguli, Hybrid stiff-string-polynomial basis functions for vibration analysis of high speed rotating beams, Comput Struct 87 (2009), 254-265.

[27] R. Attarnejad and A. Shahba, Basic displacement functions for centrifugally stiffened tapered beams, International Journal for Numerical Methods in Biomedical Engineering 27 (2009), 1385-1397.

[28] B. Yardimoglu, A novel finite element model for vibration analysis of rotating tapered Timoshenko beam of equal strength, Finite Elem Anal Des 46 (2010), 838-842.

[29] A. Shahba, R. Attarnejad and S. Hajilar, Free vibration and stability of axially functionally graded tapered Euler-Bernoulli beams, Shock and Vibration 18 (2011), 683-696.

[30] J.A. Kimball and D.E. Lovell, Variation of Young's modulus with temperature from vibration measurements, Physical Review 26 (1925), 121-124.

[31] S. Liu, Y. Zhang, Z. Du and Z. Wang, Prediction of the influence of temperature field on the critical speeds of a rod-fastened rotor, Gas Turbine Technology 2 (2011), 20-23.

[32] Ansys ANSYS help documentation, Ansys, Inc. 

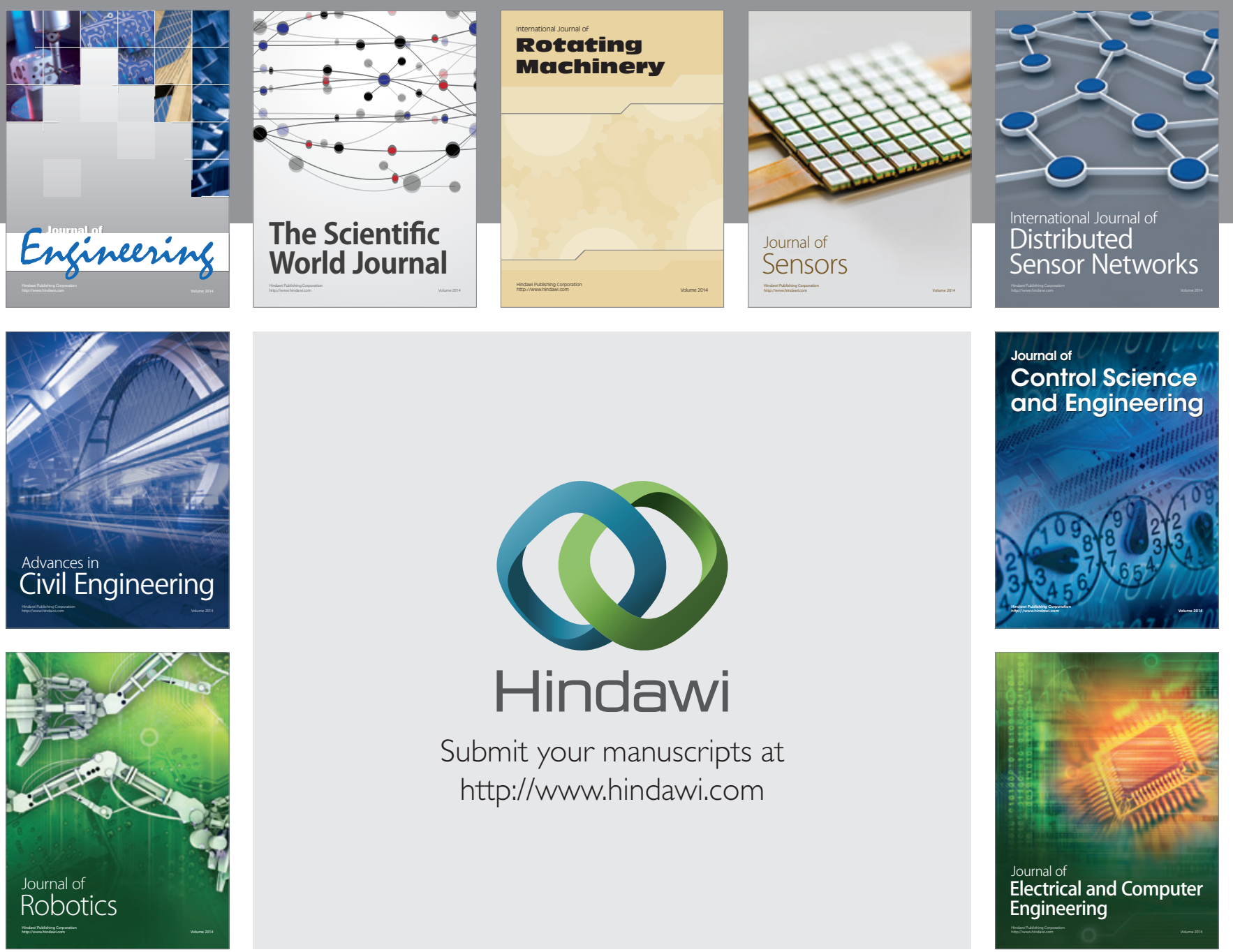

Submit your manuscripts at

http://www.hindawi.com
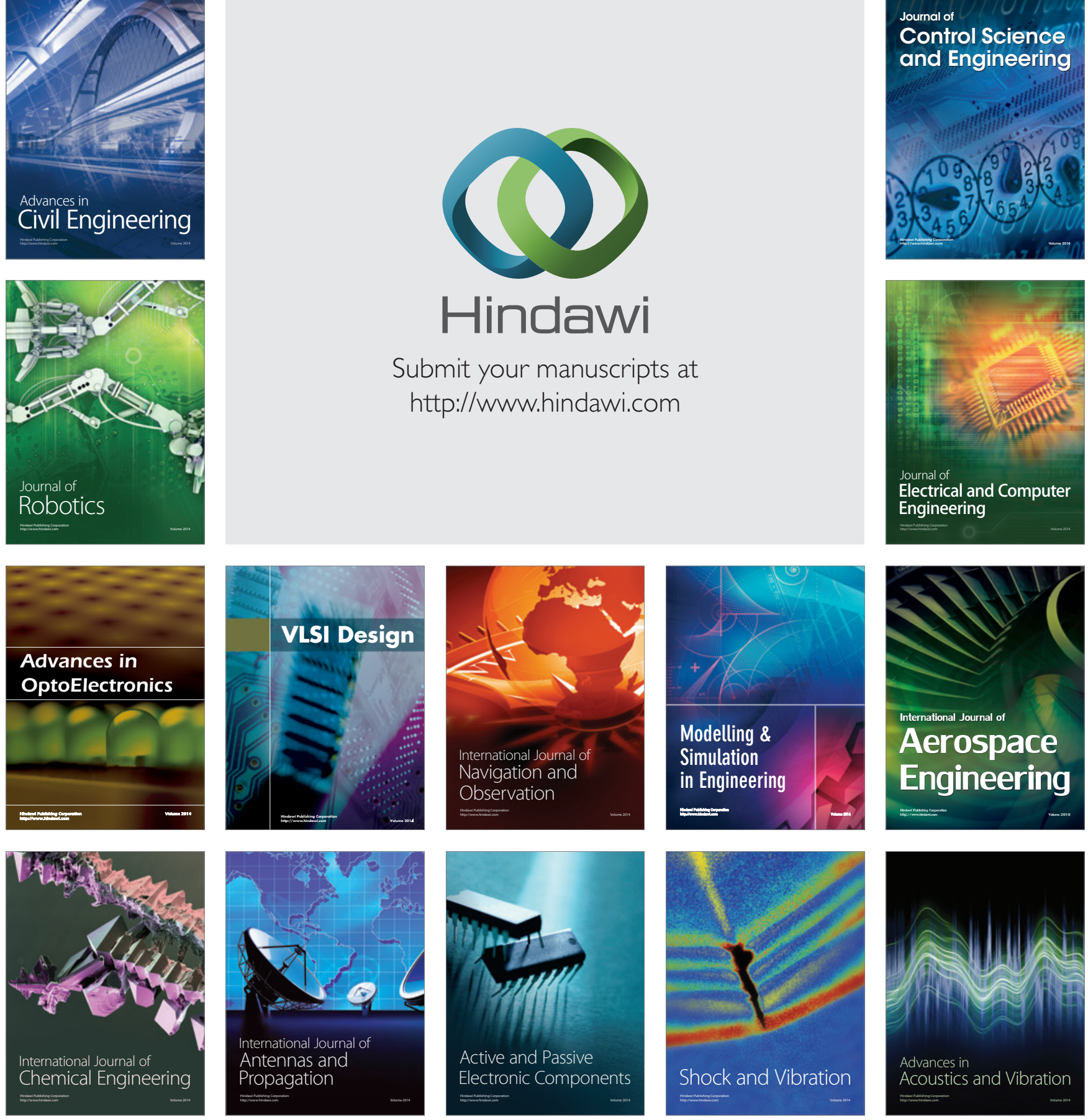